\title{
Dynamical Electroweak Symmetry Breaking with Top Quark and Neutrino Condensates
}

\author{
STEPhen P. Martin \\ Department of Physics \\ University of Florida \\ Gainesville, FL 32611
}

ABSTRACT: Recently it has been suggested that the electroweak symmetry is iroken by a top quark vacuum condensate. In that model, the prediction for the top quark mass seems to be in conflict with indirect experimental upper bounds. We propose a new scenario in which the electroweak symmetry is broken by a combination of top quark and third generation neutrino condensates, involving a right-handed gauge singlet neutrino. We show that the top quark mass comes out smaller in this model and can easily lie in the experimentally favored window. The resulting neutrino spectrum is phenomenologically acceptable if we make the natural assumption that the right handed neutrino has a very large Majorana mass.

\section{DISCLAIMER}

This report was prepared as an account of work sponsored by an agency of the United States Government. Neither the United States Government nor any agency thireof, nor any of their employees, makes any warranty, express or implied, or assumes any legal liability or responsibility for the accuracy, completeness, or usefulness of any information, apparatus, product, or process disclosed, or represents that its use would not infringe privately owned rights. Reference herein to any specific commercial product, process, or service by trade name, trademark, manufacturer, or otherwise does not necessarily constitute or imply its endorsement, recommendation, or favoring by the United States Government or any agency thereof. The views and opinions of authors expressed herein do not necessarily state or reflect those of the United States Government or any agency thereof. 


\section{Introduction}

The mass of the top quark is now known to be at least $89 \mathrm{GeV}$ from direct searches at CDF. This means that the Yukawa coupling of the top quark to the Higgs boson is at least about 15 times as strong as the bottom quark Yukawa coupling, and of course dwarfs the other standard model Yukawa couplings by even greater margins. Because of this relatively strong coupling of the top quark to the Higgs, it is natural to consider the possibility that the top quark plays an essential and unique role in breaking the electroweak symmetry by some dynamical mechanism. In this spirit, a number of authors[1-4] have proposed that the electroweak symmetry is broken, not by a fundamental Higgs scalar, but by a top quark vacuum condensate $\langle t t\rangle \neq 0$. In this "top bootstrap" model, the top quark condensate is supposed to be induced by a four-fermion interaction introduced at a scale $\Lambda$ which must be taken to be larger than the electroweak scale.

At scales far below $\Lambda$, the effective action in the top condensate model should be just the same as in the standard model, but the Higgs scalar is now a composite particle. Bardeen, Hill and Lindner [4] (hereafter referred to as BHL) pointed out that the compositeness of the Higgs should cause the usual standard model running coupling constants to have a very special behavior; the top quark Yukawa coupling $y_{t}(\mu)$ should diverge near the compositeness scale $\mu=\Lambda$, and meanwhile the running quartic Higgs self-roupling $\lambda(\mu)$ should obey $\lim _{\mu \rightarrow \Lambda} \lambda(\mu) / y_{t}(\mu)^{4}=0$. This interpretation of the compositeness of the Higgs is attractive for two reasons. First, it provides that the main tools for discovering quantitative predictions of the model are simply the usual standard model renormalization group equations; one picks out those special renormalization group trajectories which have the appropriate singular behavior near the compositeness scale. Second, one finds that the model has effectively one less parameter than the standard model. In practice, one evolves the renormalization group equations down from the singularities at the arbitrarily chosen scale $\Lambda$ to predict the values of both $y_{t}$ and $\lambda$ near the electroweak scale. Thus if $\Lambda$ is treated as an inaccessible unknown, then measurement of the top quark mass should in principle allow one to predict the mass of the Higgs. 
Unfortunately, it appears that the predictions implied by BHL's analysis are already on very shaky experimental ground. Although the top quark has not yet been directly observed, there are indirect upper bounds on its mass. According to experimental tests of the standard model, the top quark mass is most probably less than $200 \mathrm{GeV}$; a global fit to the present data for the standard model with just one Higgs doublet with a mass of $250 \mathrm{GeV}$ yields particularly stringent upper bounds on $m_{\text {top }}$ of $(157,165,180) \mathrm{GeV}$ at confidence levels of $(90,95,99)$ percent respectively[5]. (These limits can be relaxed somewhat if a heavier Higgs or a non-minimal Higgs sector is invoked.) The problem is that for the top condensate model, the values obtained by BHL for the top mass are inevitably too large. Even if the compositeness scale is taken to be as large as the Planck scale $\Lambda=10^{19} \mathrm{GeV}$, then BHL find that $m_{\text {top }}$ should be about $218 \mathrm{GeV}$, within a few percent accuracy. Moreover, as the compositeness scale $\Lambda$ is decreased, the predicted result for $m_{\text {top }}$ grows monotonically. For a GUT-scale $\Lambda=10^{15} \mathrm{GeV}$, BHL predict a top mass of about $230 \mathrm{GeV}$, while for a much lower compositeness scale like $\Lambda=10^{4} \mathrm{GeV}$, the top mass prediction becomes a clearly unacceptable $450 \mathrm{GeV}$.

There is actually a simple way to see why $m_{\text {top }}$ is necessarily so large in the top quark condensate model[4]. The renormalization group equation which governs the running of the top quark Yukawa coupling in the standard model is (to one loop order)

$$
\begin{aligned}
16 \pi^{2} \frac{d}{d t} y_{t} & =y_{t}\left[\frac{9}{2} y_{t}^{2}-8 g_{3}^{2}-\frac{9}{4} g_{2}^{2}-\frac{17}{12} g_{1}^{2}\right] \\
t & =\log \left(\mu / M_{Z}\right)
\end{aligned}
$$

where we have ignored the negligible contributions of the other Yukawa couplings. According to the interpretation of BHL, the compositeness of the Higgs implies that we should take $\lim _{\mu \rightarrow \Lambda} y_{t}(\mu)=\infty$ as a boundary condition and run eq. (1.1) down to predict $y_{t}(\mu)$ for $\mu$ near the electroweak scale. The important feature of (1.1) is that the small $\mu$ behavior of $y_{t}$ is: governed by the existence of an infrared quasi-fixed point [6,7] when the RHS vanishes. Since the $S U(2)$ and $U(1)_{Y}$ couplings make a small contribution, we can expect that the Yukawa coupling will end up somewhere near

$$
y_{t} \approx 4 g_{3} / 3
$$


This leads to a rough prediction of the top mass which is insensitive to the choice of compositeness scale. Using $m_{t}=y_{t} 246 \mathrm{GeV} / \sqrt{2}$ and the experimental result $[8] g_{3}\left(M_{z}\right)^{2}=$ $1.48 \pm .10$, one finds that $m_{\text {top }} \approx 280 \mathrm{GeV}$. Of course, if we choose $\Lambda$ to be sufficiently small, then $y_{t}$ will not have sufficient running room between the electroweak breaking scale and $\Lambda$ to reach the quasi-fixed point, and it is easy to see that this leads to a larger prediction for $m_{\text {top. }}$. Conversely, if $\Lambda$ is taken to be sufficiently large, then $y_{t}$ will see a smaller $S U(3)_{c}$ running coupling constant $g_{3}$ at larger scales, and so the top mass can come out slightly smaller.

Assuming that the experimental upper bound on the top quark mass is indeed valid, it appears to be necessary to introduce some radical modification of the top quark condensate model in order to save it. Now, it is unlikcly that any modification of the model in the desert between the electroweak scale and the compositeness scale $\Lambda$ can do much good, because the essential reason for the large top mass prediction does not lie in the desert, but at the IR quasi-tixed print (1.2), which is independent of physics at higher scales.

Let us consider instead the possibility that some other fermion "shares the burden" of $S U(2) \times U(1)_{Y}$ symmetry breaking with the top quark. If some other fermion acquires a condensate in the same manner as the top quark, then we might hope that the coupled noninear renormalization group equations governing the running of the two relevant Yukawa couplings would end up providing for a smaller $y_{t}$ at the electroweak scale and thus a smaller $m_{\text {top. }}$. Clearly, the other Yukawa coupling participating in this cannot be any of the ones already present in the standard model. The reason is simply that in order for the other: Yukawa coupling to be large enough to affect $y_{t}$ in a significant way, it would have to also endow its fermion with a mass significantly higher than the known experimental values. Put another way, even the bottom quark has a negligible effect on the running of $y_{t}$, and on the fixed point (1.2).

One possibility is that there is a fourth generation of fermions which have a mass greater than the top. This seems a bit ad hoc, since it abandons the original motivation based on the strength of the top quark coupling to the Higgs. Furthermore, one must 
be careful to avoid violating the experimental limit of three neutrino species lighter than $M_{Z} / 2$ given us by LEP and SLC. Neverthless, it has been shown [9] that such a model is viable; the fermion condensates induce a composite Majoron as well as a composite Higgs, and the fourth generation neutrino can naturally acquire a large mass. Other possible variations on the original top condensate model have appeared in refs. [10-14].

In this paper, we will investigate instead the possibility that the electroweak symmetry is broken by a combination of top quark and neutrino condensates in a three generation model. In addition to the usual quarks and leptons of the standard model, we suppose that there are right-handed neutrinos $N_{e, \mu, \tau}$ for each generation. These are taken to be singlets under the standard model gauge group $S U(2)_{c} \times S U(2) \times U(1)_{Y}$. We wish to explore the idea that the third generation right handed neutrino has a Yukawa coupling $y_{N}$ to the Higgs and the usual left-handed third generation lepton doublet $L=\left(\nu_{\tau} \tau\right)$ whose strength is just comparable to that of top quark Yukawa coupling $y_{t}$. We further suppose that there is an appropriate set of four-fermi interactions at some large scale $\Lambda$ which will produce condensates $\langle\bar{t} t\rangle \neq 0$ and $\langle\bar{\nu} N\rangle \neq 0$. The electroweak symmetry is then spontaneously broken by both condensates, with the top quark obtaining a smaller mass than in the original top condensate model, as we shall see.

In order for the third generation neutrino to be effective in lowering the top mass, its Yukawa coupling must endow it with a Dirac mass on the order of $100 \mathrm{GeV}$ or so. This is not a disaster, however, because it is natural to assume that the see-saw mechanism [15] will determine the neutrino mass eigenstates. Because the right-handed neutrino $N$ is a gauge singlet in the standard model, it is eminently reasonable that it should acquire a large bare Majorana mass $M$. Then the third generation neutrino mass matrix will take the form

$$
\left(\begin{array}{cc}
0 & m \\
m & M
\end{array}\right)
$$

where we assume that, very roughly speaking, $m \approx 100 \mathrm{GeV}$. Now if the Majorana mass $M$ is taken to be of the GUT scale, e.g. $M \approx 10^{15} \mathrm{GeV}$, then the eigenvalues of (1.3) are approximately $m_{\text {light }} \approx m^{2} / M \approx 10^{-2} \mathrm{eV}$ and $m_{\text {heavy }} \approx M \approx 10^{15} \mathrm{GeV}$. The heavy 
neutrino mass eigenstate thus acquires a GUT-scale mass and has a negligible effect on low energy physics, while the light ne:urrino mass eigenstate acquires a mass well below the experimental limits. In fact, a neutrino mass eigenstate with $m_{\text {light }} \approx 10^{-2} \mathrm{eV}$ fits in very nicely with the notion that the deficit of observed solar neutrinos can be explained by the MSW effect[16], if we assume a moderate mixing between the third generation neutrino and its first and second generation cousins. Roughly speaking, a non-adiabatic MSW explanation of the solar neutrino deficit can work if the electron neutrino and muon neutrino masses satisfy[17]

$$
\left(m_{\nu_{\mu}}^{2}-m_{\nu_{e}}^{2}\right) \sin ^{2} \theta \approx 10^{-8} \mathrm{eV}^{2}
$$

where $\theta$ is the mixing angle between the two species, and the masses $m_{\nu_{e}}$ and $m_{\nu_{\mu}}$ arise from $m_{\text {light }}$ by mixing with the third generation. (Such a mixing can arise e.g. from a non-diagonal matrix of bare Majorana masses for $N_{e, \mu, \tau}$.) Thus we conclude that a third generation neutrino with a Dirac mass of order $100 \mathrm{GeV}$ is not only acceptable, but may actually be phenomenologically desireable. This is clearly a very elastic conclusion given the paucity of experimental data; a large range of possibilities for the bare Majorana mass $M$ is available depending on the details of the mixing between the three neutrino species.

Why should the top quark Yukawa coupling and the third generation neutrino Yukawa coupling be comparable? We will here regard this as simply an ad hoc assumption of the model. However, it is worth noting than in certain GUT models (e.g. $S O(10)$ ), one obtains the tree-level prediction $y_{t}=y_{N}$ at the GUT breaking scale, due to the fact that the top quark and the neutrino inhabit the same fermion multiplet before symmetry breaking. This relation is of course modified by loop effects in passing to lower energy scales.

\section{A Model with Top Quark and Neutrino Condensates}

Let us consider the usual standard model action without a fundamental Higgs scalar, and add in four-fermion couplings as follows:

$$
L_{\Lambda}=L_{\text {usual }}+\left(\kappa_{t} \bar{Q}^{i} t+\kappa_{N} \bar{L}^{i} N\right)\left(\kappa_{t} \bar{t} Q_{i}+\kappa_{N} \bar{N} L_{i}\right)
$$


Here $\kappa_{t}$ and $\kappa_{N}$ are coupling constants with dimensions of (mass) ${ }^{-1}, Q_{i}=(t b)$ is the left-handed quark doublet, and $L_{i}=\left(\nu_{\tau} \tau\right)$ is the left-handed lepton doublet. The index $i=1,2$ is an $S U(2)$ index and the $S U(3)$ color indices are suppressed. $L_{\Lambda}$ is postulated to be the effective action at the compositeness scale $\Lambda$. We can rewrite (2.1) by introducing an auxiliary, static, scalar field $H_{i}$ with the same quantum numbers as the usual Higgs doublet:

$$
L_{\Lambda}=L_{\text {usual }}+\left(H_{i}\left[Y_{t} \bar{Q}^{i} t+Y_{N} \bar{L}^{i} N\right]+\text { h.c. }\right)-M_{H}^{2} H^{\dagger} H
$$

where $Y_{t}$ and $Y_{N}$ are Yukawa type couplings with $Y_{t}=\kappa_{t} M_{H}$ and $Y_{N}=\kappa_{N} M_{H}$. Now following the philosophy of BHL, we can imagine computing the effective action at scales $\mu \ll \Lambda$ by integrating out the modes of all fields with Euclidean momenta in the range $\mu^{2}<$ $p_{E}^{2}<\Lambda^{2}$. This will induce in the resulting low energy effective theory all renormalizable and gauge invariant terms involving the field $H_{i}$, including, besides the types of terms already in (2.2), a kinetic term and a quartic self-coupling for $H_{i}$. So one obtains, for $\mu \ll \Lambda$, the low energy effective action

$$
\begin{aligned}
L(\mu)=L_{\text {usual }}(\mu) & +Z(\mu)\left|D_{\mu} H\right|^{2}-\widetilde{m}_{H}^{2}(\mu) H^{\dagger} H-\frac{\tilde{\lambda}(\mu)}{2}\left(H^{\dagger} H\right)^{2} \\
& +\left(H_{i}\left[\tilde{y}_{t}(\mu) \bar{Q}^{i} t+\widetilde{y}_{N}(\mu) \bar{L}^{i} N\right]+\text { h.c. }\right)
\end{aligned}
$$

All of the coupling constants in the low energy effective action run with the scale $\mu$ according to Wilson's renormalization group equations.

By comparison of (2.3) with (2.2), we obtain the following boundary conditions on the behavior $\mathrm{c} f$ the running couplings at the scale $\mu=\Lambda$;

$$
\begin{array}{lrl}
Z(\Lambda)=0 ; & \tilde{\lambda}(\Lambda)=0 & \\
\tilde{y}_{t}(\Lambda)=Y_{t} ; & \tilde{y}_{N}(\Lambda)=Y_{N} ; & \widetilde{m}_{H}^{2}(\Lambda)=M_{H}^{2} .
\end{array}
$$

Now (2.3) is just the usual action for the standard model, except that the kinetic term for $H$ does not have the standard (unit) normalization. To rectify this, one can renormalize the field $H$ according to $H \rightarrow H / \sqrt{Z(\mu)}$ and reexpress the action in terms of the rescaled parameters

$$
y_{t}=\tilde{y}_{t} / \sqrt{Z} \quad y_{N}=\tilde{y}_{N} / \sqrt{Z}
$$




$$
m_{H}^{2}=\tilde{m}_{H}^{2} / Z \quad \lambda=\tilde{\lambda} / Z^{2}
$$

Then the effective action takes precisely the same form as the standard model:

$$
\begin{aligned}
L(\mu)=L_{\text {usual }}(\mu) & +\left|D_{\mu} H\right|^{2}-m_{H}^{2}(\mu) H^{\dagger} H-\frac{\lambda(\mu)}{2}\left(H^{\dagger} H\right)^{2} \\
& +\left(H_{i}\left[y_{t}(\mu) \bar{Q}^{i} t+y_{N}(\mu) \bar{L}^{i} N\right]+\text { h.c. }\right)
\end{aligned}
$$

(We should note that (2.1) contains only two independent parameters $\kappa_{t}$ and $\kappa_{N}$, while a more general possibility might contain the terms $\bar{Q}^{i} t \bar{t} Q_{i}$ and $\bar{L}^{i} N \bar{N} L_{i}$ and $\bar{Q}^{i} t \bar{N} L_{i}+$ h.c. with three independent coefficients. This would correspond in general to the more complicated situation of two composite Higgs doublets. We will not investigate that possibility here.)

The boundary conditions (2.4) translate into conditions on the standard model parameters appearing in (2.6):

$$
\begin{aligned}
& \lim _{\mu \rightarrow \Lambda} y_{t}(\mu)=\infty \quad \lim _{\mu \rightarrow \Lambda} y_{N}(\mu)=\infty \\
& \lim _{\mu \rightarrow \Lambda} \lambda(\mu) / y_{t}(\mu)^{4}=\lim _{\mu \rightarrow \Lambda} \lambda(\mu) / y_{N}(\mu)^{4}=0 \\
& \lim _{\mu \rightarrow \Lambda} y_{t}(\mu) / y_{N}(\mu)=Y_{t} / Y_{N} \\
& \lim _{\mu \rightarrow \Lambda} m_{H}^{2}(\mu) / y_{N}(\mu)^{2}=M_{H}^{2} / Y_{N}^{2}
\end{aligned}
$$

So in this model, the compositeness of the Higgs scalar manifests itself as the boundary conditions (2.7)-(2.10) on the running coupling constants in the standard model.

In order to translate the compositeness boundary conditions (2.7)-(2.10) into low energy predictions of the model, one can follow the strategy used by BHL and evolve the couplings from the scale $\Lambda$ down to the electroweak scale using the one loop renormalization group equations

$$
\begin{aligned}
16 \pi^{2} \frac{d}{d t} y_{t} & =y_{t}\left(\frac{9}{2} y_{t}^{2}+y_{n}^{2}-8 g_{3}^{2}-\frac{9}{4} g_{2}^{2}-\frac{17}{12} g_{1}^{2}\right) \\
16 \pi^{2} \frac{d}{d t} y_{N} & =y_{N}\left(3 y_{t}^{2}+\frac{5}{2} y_{n}^{2}-\frac{9}{4} g_{2}^{2}-\frac{3}{4} g_{1}^{2}\right) \\
16 \pi^{2} \frac{d}{d t} \lambda & =12 \lambda^{2}+\lambda\left(12 y_{t}^{2}+4 y_{N}^{2}-3 g_{1}^{2}-9 g_{2}^{2}\right)-12 y_{t}^{4}-4 y_{N}^{4}+\frac{3}{4} g_{1}^{4}+\frac{3}{2} g_{1}^{2} g_{2}^{2}+\frac{9}{4} g_{2}^{4} .
\end{aligned}
$$


The standard model $S U(3)_{c}, S U(2)$ and $U(1)_{Y}$ gauge coupling constants appearing in (2.11) run according to the renormalization group equations, which at one loop order are

$$
\begin{aligned}
16 \pi^{2} \frac{d}{d t} g_{1} & =41 g_{1}{ }^{3} / 6 \\
16 \pi^{2} \frac{d}{d t} g_{2} & =-19 g_{2}{ }^{3} / 6 \\
16 \pi^{2} \frac{d}{d t} g_{3} & =-7 g_{3}{ }^{3}
\end{aligned}
$$

We use the values $g_{1}\left(M_{Z}\right)^{2}=.128, g_{2}\left(M_{Z}\right)^{2}=.423$, and $g_{3}\left(M_{Z}\right)^{2}=1.48$ and $M_{Z}=$ $91.17 \mathrm{GeV}$.

As one approaches the scale $\Lambda$, the top quark and Yukawa couplings become large. Thus the one loop beta functions for $y_{t}$ and $y_{N}$ are very large and positive, but the higher loop contributions also begin to become important. Furthermore, irrelevant operators in the effective action which are suppressed at lower scales can have an important effect near $\Lambda$. It is important to realize that this makes it impossible to make precise statements about the behavior of the theory near the compositeness scale using just the perturbative beta functions to any finite order in the loop expansion. In particular, there is no way to be certain that the divergence of the Yukawa couplings as one approaches $\Lambda$ is really maintained in the full nonperturbative theory. In practice, one just has to assume that the rapid growth of $y_{t}$ and $y_{N}$ near $\Lambda$ can be extrapolated in a qualitative sense. In other words, we trust that once the Yukawa couplings become sufficiently large that the one loop beta functions can no longer be trusted, they will nevertheless continue to diverge as $\mu \rightarrow \Lambda$. A necessary consequence of this is that we should not attach any physical significance to the details of the behavior of the couplings, such as the ratios (2.9)-(2.10), near $\Lambda$. Instead, the detailed behavior of the couplings (as calculated from the one loop beta functions) near $\Lambda$ should merely be viewed as a paran. atrization of our ignorance of the true behavior of tise theory near $\Lambda$. What saves the day is that the behavior of the theory at much lower scales is relatively insensitive to the unknown behavior near the scale $\Lambda$.

Once we have evolved the parameters $y_{t}(\mu), y_{N}(\mu)$ and $\lambda(\mu)$ down from $\Lambda$ to the electroweak scale, we can predict the value of the top quark mass according to the mass 
shell condition

$$
m_{\text {top }}=y_{t}\left(\mu=m_{\text {top }}\right) v / \sqrt{2}
$$

with $v=246 \mathrm{GeV}$. The Dirac mass parameter of the neutrino at the scale $\mu=M_{Z}$ is similarly given by

$$
m_{\text {neutrino }}=y_{N}\left(\mu=M_{Z}\right) v / \sqrt{2}
$$

Finally, the mass of the composite Higgs is predicted to be

$$
m_{\mathrm{Higgs}}=\lambda\left(\mu=m_{\mathrm{Higgs}}\right)^{1 / 2} v
$$

In practice, it turns out to be convenient to carry out the numerical running of the couplings in terms of the quantities

$$
z_{t} \equiv 1 / y_{t}^{2}, \quad z_{N} \equiv 1 / y_{N}^{2}
$$

and

$$
h_{N} \equiv \lambda / y_{N}^{4} \quad \text { or } \quad h_{t} \equiv \lambda / y_{t}^{4}
$$

instead of the couplings themselves. (It turns out to be numerically convenient to use the variable $h_{N}$ in situations where $z_{N} \leq z_{t}$ near $\Lambda$ and to use the variable $h_{t}$ if $z_{t} \leq z_{N}$ near

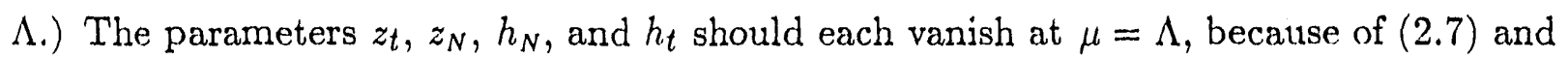
(2.8), and by using (2.11) we find that to one loop order they run according to:

$$
\begin{aligned}
16 \pi^{2} \frac{d}{d t} z_{t} & =-9-2 \frac{z_{t}}{z_{N}}+z_{t}\left(16 g_{3}^{2}+\frac{9}{2} g_{2}^{2}+\frac{17}{6} g_{1}^{2}\right) \\
16 \pi^{2} \frac{d}{d t} z_{N} & =-5-6 \frac{z_{N}}{z_{t}}+z_{N}\left(\frac{9}{2} g_{2}^{2}+\frac{3}{2} g_{1}^{2}\right) \\
16 \pi^{2} \frac{d}{d t} h_{N}= & -4-6 \frac{h_{N}}{z_{N}}+12 \frac{h_{N}^{2}}{z_{N}^{2}}-12 \frac{z_{N}^{2}}{z_{t}^{2}}+z_{N}^{2}\left(\frac{3}{4} g_{1} 4+\frac{3}{2} g_{1}^{2} g_{2}{ }^{2}+\frac{9}{4} g_{2}{ }^{4}\right) \\
16 \pi^{2} \frac{d}{d t} h_{t}= & -12-6 \frac{h_{t}}{z_{t}}+12 \frac{h_{t}^{2}}{z_{t}^{2}}-4 \frac{z_{t}^{2}}{z_{N}^{2}}+z_{t}^{2}\left(\frac{3}{4} g_{1}^{4}+\frac{3}{2} g_{1}^{2} g_{2}^{2}+\frac{9}{4} g_{2}^{4}\right) \\
& +\frac{8}{3} h_{t} g_{1}^{2}+32 h_{t} g_{3}^{2}
\end{aligned}
$$

Now, for each value of $\Lambda$, we proceed by taking $h_{N}(\Lambda)=0\left(\right.$ or $\left.h_{t}(\Lambda)=0\right)$ and picking very small values for $z_{t}(\Lambda)$ and $z_{N}(\Lambda)$, and numerically integrating the equations (2.18)-(2.2!) 
down to the electroweak scale. The choice of very small values at $\Lambda$ is just a numerical convenience; it corresponds to the freedom to pick the ratio of limits in (2.9). One can also pick a small value for $h_{N}(\Lambda)$ (or $h_{t}(\Lambda)$ ), but the results turn out to be extremely insensitive to this, so we just take $h_{N}(\Lambda)=0$ ( or $h_{t}(\Lambda)=0$ ). (This strong insensitivity of the low energy results to $h_{N}(\Lambda)$ or $h_{t}(\Lambda)$ can be understood from the fact that the running of $h_{N}$ and $h_{t}$ is determined completely by the gauge couplings and $z_{N}$ and $z_{t}$, which do not in turn depend on $h_{N}$ or $h_{t}$ at all to one loop order.) So the resulting predictions for $m_{\text {top }}$, $m_{\text {Higgs }}$ and the neutrino Dirac neutrino mass parameter $m_{\text {neutrino }}$ can then be thought of as functions only of the scale $\Lambda$ and of the ratio of the initial very small values chosen for $z_{t}(\Lambda)$ and $z_{N}(\Lambda)$. However, as we have already mentioned, the specific detailed behavior such as the numerical ratios of the couplings near $\Lambda$ should not be taken seriously except as a parametrization of our ignorance concerning the true behavior near the compositeness scale. Thus for the purposes of presenting the results, it is much more meaningful to replace the ratio of the very small initial values of $z_{t}(\Lambda)$ and $z_{N}(\Lambda)$ as a parametrization of our ignorance, by a suitable low energy parameter which varies smoothly as the high energy boundary conditions are varied. A good choice for this "ignorance parameter" is $m_{\text {neutrino }}$. Thus we present our results by giving $m_{\text {top }}$ and $m_{\text {Higgs }}$ as a function of the two parameters $\Lambda$ and $m_{\text {neutrino. }}$

The numerical predictions for various choices of $\Lambda$ ranging from the Planck scale down to $\Lambda=10^{4} \mathrm{GeV}$ are given in Figs. 1-5. It is apparent that as $m_{\text {neutrino }}$ is increased, the mass of the top quark decreases in this model. One way of understanding this qualitatively is that $y_{t}$ and $y_{N}$ are in competition with each other in the terms in parentheses in eqs. (2.11). For small values of $m_{\text {neutrino, }}$, the effect of the neutrino should become negligible. As we take $m_{\text {neutrino }}$ to zero in Figs. 1-5 we indeed recover the results given by BHL, as expected. On the other hand, if we assume that the neutrino Dirac mass parameter is sufficiently large, the top quark mass can be made arbitrarily small. In particular, it is not difficult to make $m_{\text {top }}$ lie in the experimentally favored window. For our scheme to work, we need to assume that the two relevant Yukawa couplings should be roughly of the same order. The prediction for the mass of the Higgs is evidently roughly insensitive to the value of 
$m_{\text {neutrino, }}$ and increases slowly as $\Lambda$ decreases.

One feature of this model is that reasonable values for the top quark mass are evidently available regardless of the choice of the compositeness scale. This might give us a way to circumvent the usual fine-tuning problem which occurs when the scale of new physics $\Lambda$ is taken to be much greater than the electroweak breaking scale. However, it is important to realize that when $\Lambda$ is taken small enough to alleviate the fine-tuning problem, the reliability of predictions made using the renormalization group becomes suspect. (The fine tuning associated with large values of $\Lambda$ is actually beneficial from the point of view of making accurate predictions, because it provides a lot of "running room" for the coupling constants, which tends to wash out unknown details of the theory near $\Lambda$.) Still, this is an indictment of our method of calculation, and not necessarily of the model itself. So one may hope that the qualitative features of the model will survive, and look for a more reliable scheme for calculating the consequences of the model for small $\Lambda$.

\section{Conclusion}

We have shown that a right handed third generation neutrino can successfully conspire with the top quark to break the electroweak symmetry, without giving the top quark an unacceptably large mass. The essential point is that a large Yukawa coupling for the neutrino can have a dramatic effect on the IR quasi-fixed point (1.2), with the resulting neutrino Dirac mass being hidden by the see-saw mechanism. Two of the nice features of this are that the neutrino mass eigenstates can easily obtain the right sort of masses to explain the solar neutrino deficit using the MSW effect, and that it may be possible to talse the scale $\Lambda$ to be small enough to alleviate the usual fine-tuning problem. An outstanding weakness of all models of this type is that so far they exist only as effective theories below the compositeness scale. It would be very interesting to understand how this type of mechanism could be realized as the low energy limit of some renormalizable theory. 
I am grateful to Pierre Ramond, Soo-Jong Rey, and David G. Robertson for helpful comments. This work was supported in part by the Institute for Fundamental Theory and by DOE contract DE-FG05-86-ER40272.

\section{References}

1. Y. Nambu, "BCS Mechanism, Quasi-Supersymmetry, and Fermion Mass Matrix", EFI preprint 88-39, 1988; "Quasi-Supersymmetry, Bootstrap Symmetry Breaking, and Fermion Masses", (appears in 1988 International Workshop on New Trends in Strong Coupling Gauge Theories, Nagoya, Japan, eds. Bando, Muta, and Yamawaki), EFI preprint 88-62, 1988; "Bootstrap Symmetry Breaking in Electroweak Unification", EFI preprint 89-08, 1989.

2. V. Miransky, M. Tanabashi, and K. Yamawaki, Phys. Lett. B221, 117, (1989); Mod. Phys. Lett. A 4, 1043, (1989).

3. W. Marciano, Phys. Rev. Lett. 62, 2793, (1989).

4. W. A. Bardeen, C. T. Hill, and M. Lindner Phys. Rev. D41, 1647, (1990).

5. P. Langacker and M. Luo, "Implications of Precision Electroweak Experiments for $m_{t}, \rho_{0}, \sin ^{2} \theta_{W}$, and Grand Unification", University of Pennsylvania preprint UPR0466T, February 1991; and references therein.

6. B. Pendleton and G. Ross, Phys. Lett. 98B, 291, (1981); C. T. Hill, Phys. Rev. D24, $691,(1981)$.

7. J. Kubo, K. Sibold, and W. Zimmerman, Phys. Lett. 220B, 185, (1989); Nucl. Phys. B259, 331, (1985).

8. S. Bethke, "Experimental Overview of Jet Physics and Tests uf QCD", CERN preprint. 
CERN-PPE/91-36, February 1991; and references therein.

9. C. T. Hill, M. A. Luty, and E. A. Paschos; "Electroweak Symmetry Breaking by Fourth Generation Condensates and the Neutrino Spectrum", EFI preprint EFI-9043, October 1990.

10. T. E. Clark, S. T. Love, and W. A. Bardeen, Phys. Lett. B237, 235, (1990).

11. P. Kaus and S. Meshkov, Phys. Rev. D42, 1863, (1990).

12. M. Bando, T. Kugo, N. Maekawa, N. Sasakura, Y. Watabiki, and K. Suehiro, Phys. Lett. B246, 466, (1990).

13. M. A. Luty, Phys. Rev. D41, 2893, (1900).

14. M. Suzuki, Phys. Rev. D41, 3457, (1990); Mod. Phys. Lett. A5, 1205, (1990).

15. M. Gell-Mann, P. Ramond, and R. Slansky, in Supergravity, North Holland Pub., 1979; T. Yanagida, in Proc. of the Workshop on Unified Theories and Baryon Number in the Universe, KEK, Japan, 1979.

16. S. P. Mikheyev and A. Y. Smirnov, Sov. J. Nucl. Phys. 42, 1441, (1986); L. Wolfenstein, Phys. Rev. D17, 2369, (1979).

17. S. P. Rosen and J. M. Gelb, Phys. Rev. D39, 3190, (1989); J. N. Bahcall and H. A. Bethe, Phys. Rev Lett. 65, 2233, (1990).

Figure Captions

Figures 1-5: Predictions for the masses of the top quark (circles) and composite Higgs scalar boson (squares) are shown as a function of the neutrino Dirac mass parameter for choices of compositeness scale $\Lambda=10^{19} \mathrm{GeV}, 10^{15} \mathrm{GeV}, 10^{11} \mathrm{GeV}, 10^{8} \mathrm{GeV}$, and $10^{4} \mathrm{GeV}$. 


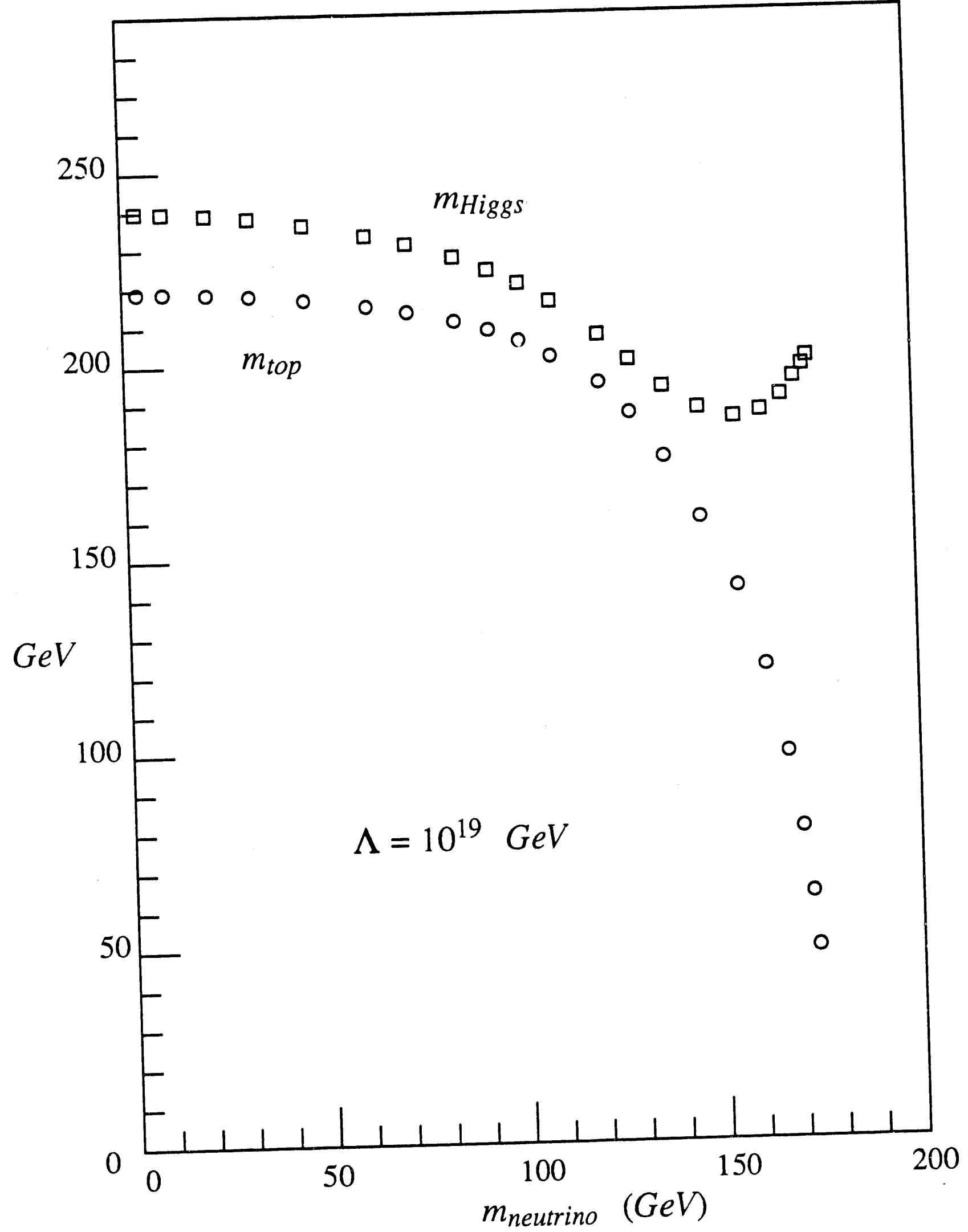

Fig. 1 


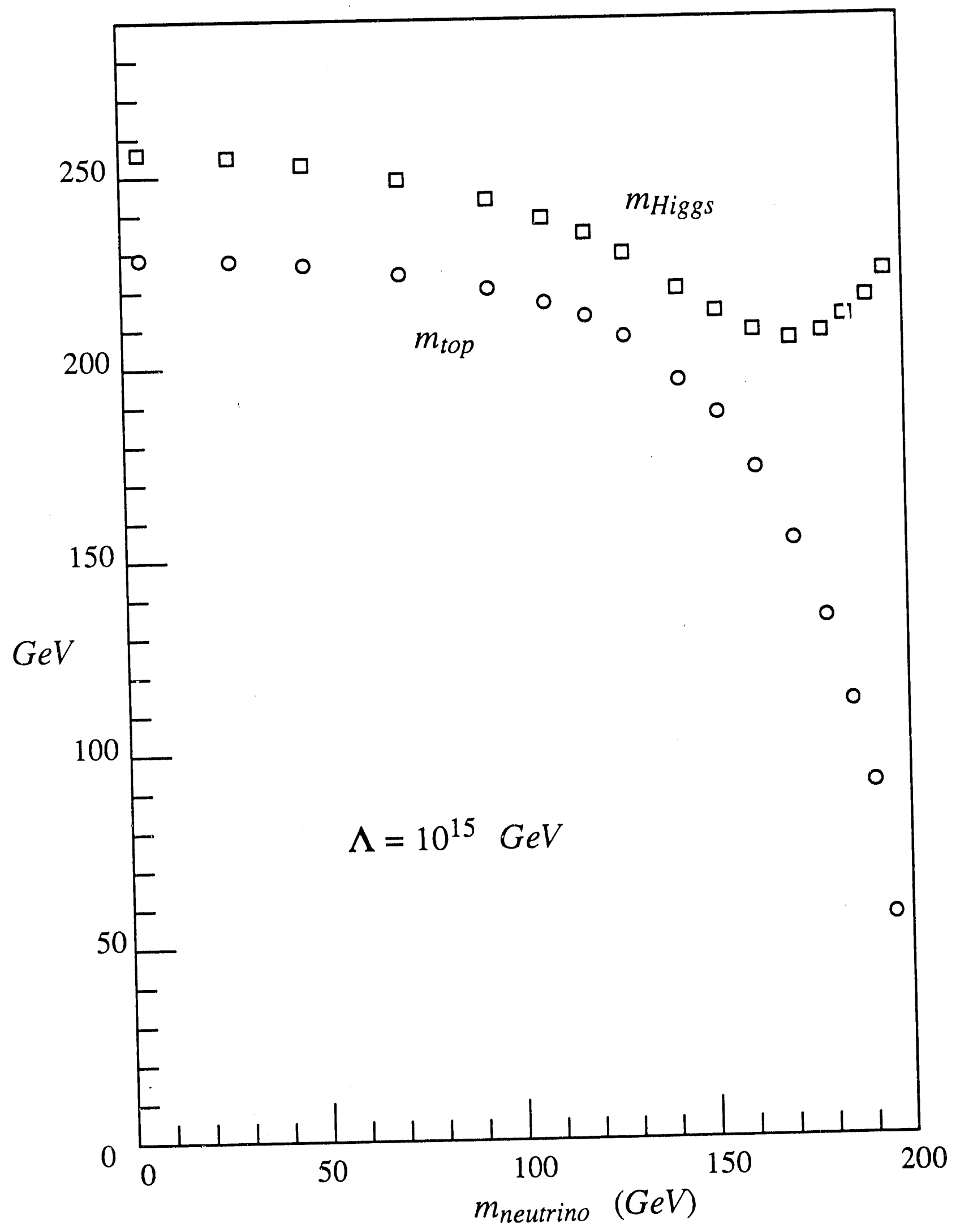

Fig. 2 


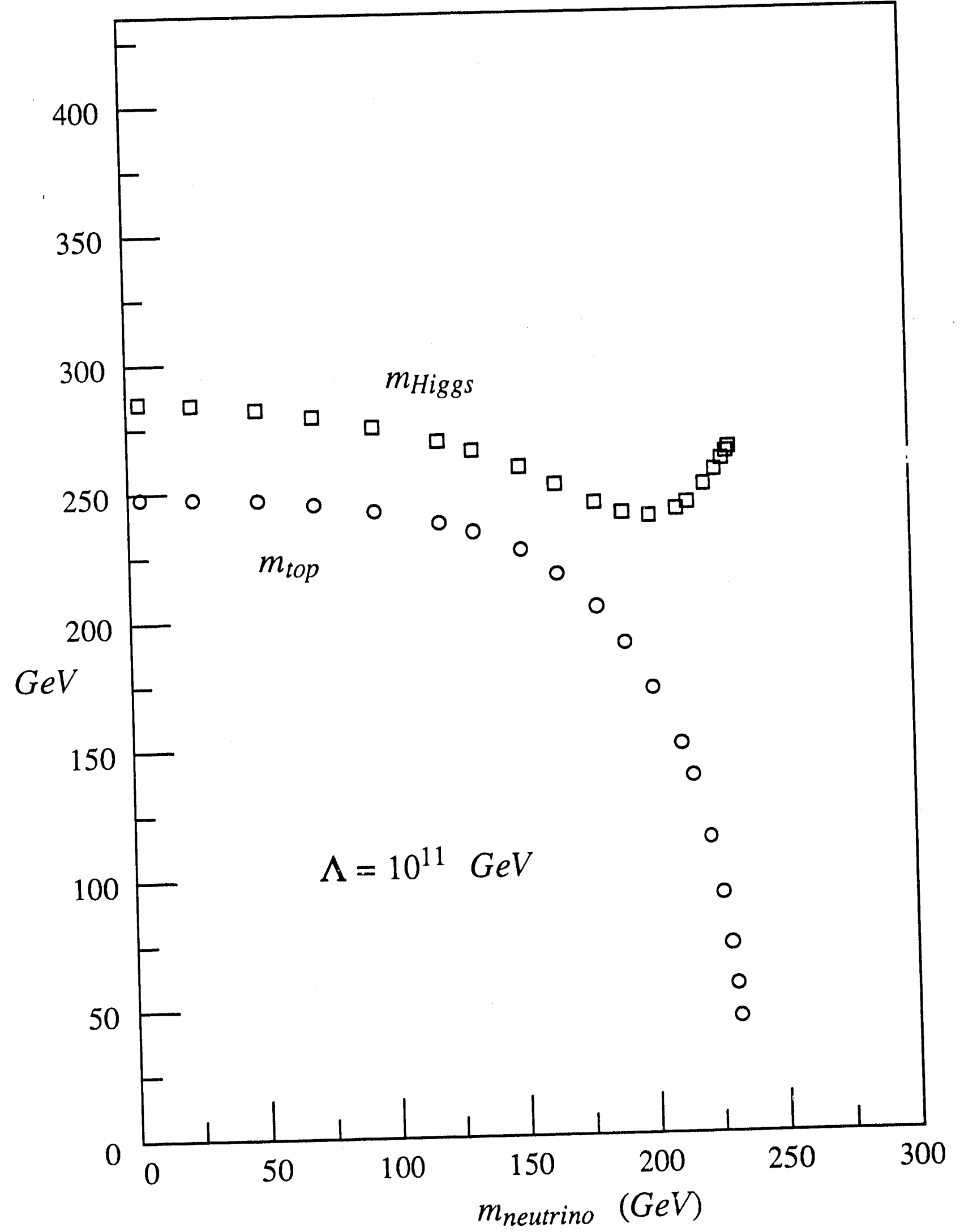

Fig. 3 


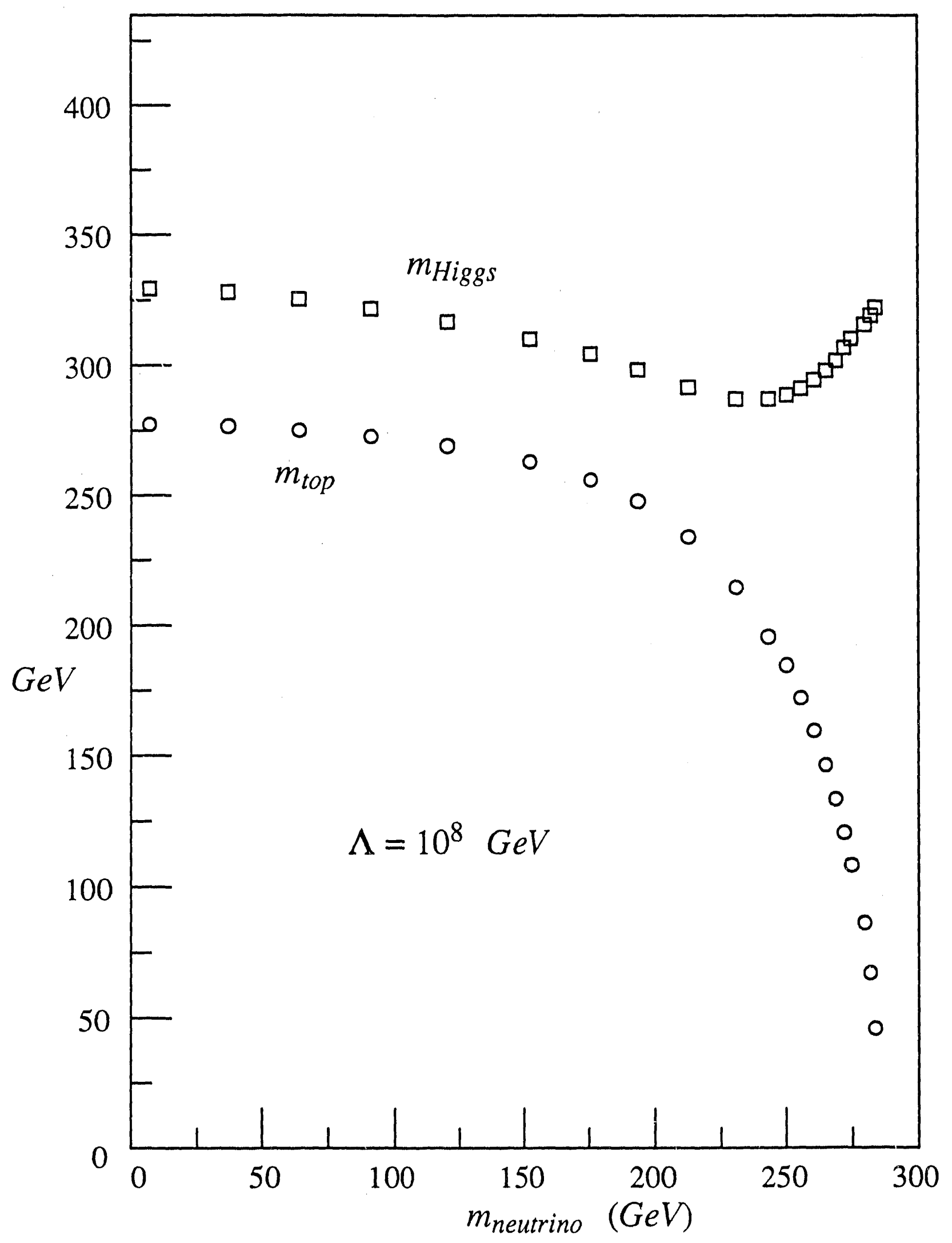

Fig. 4 


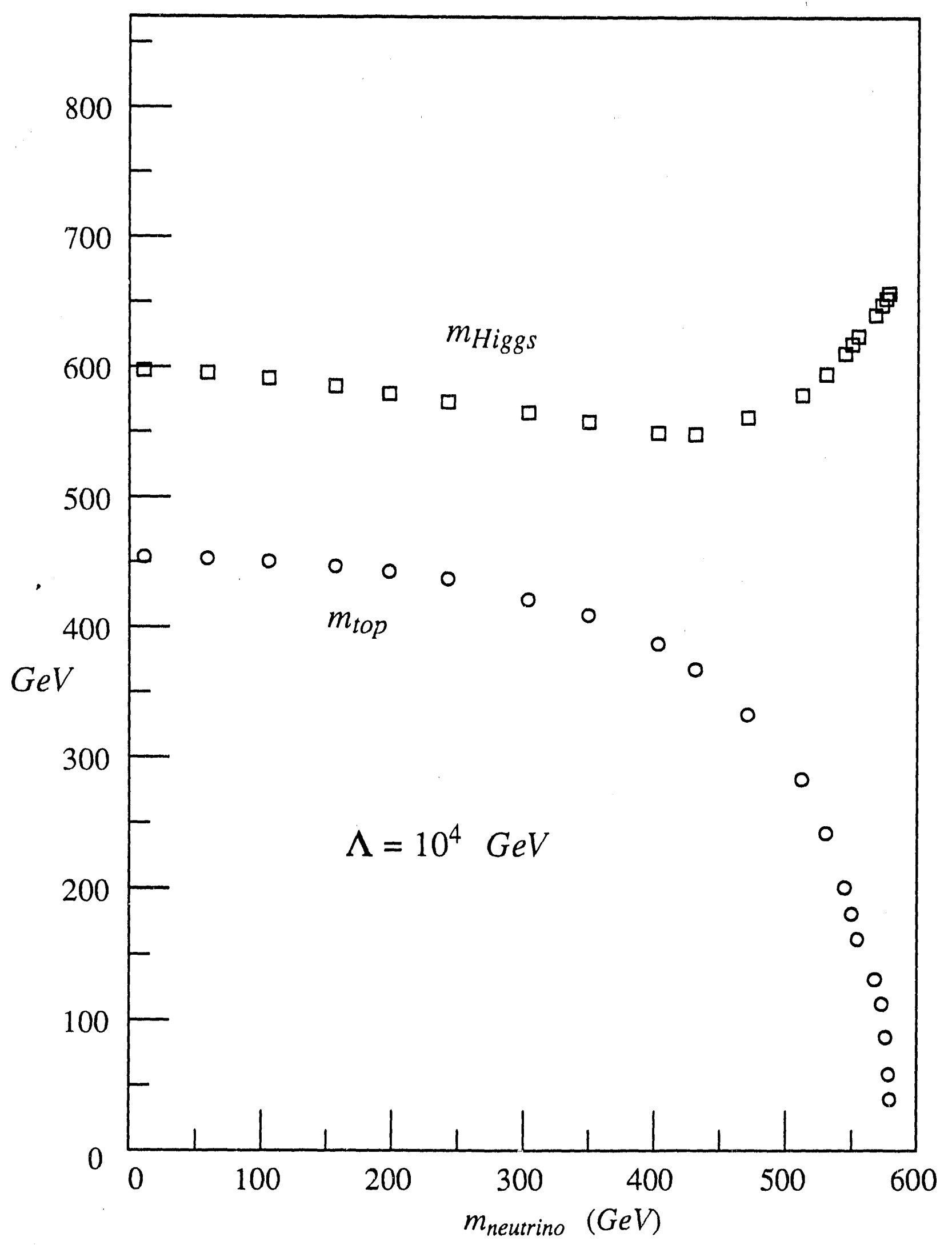

Fig. 5 


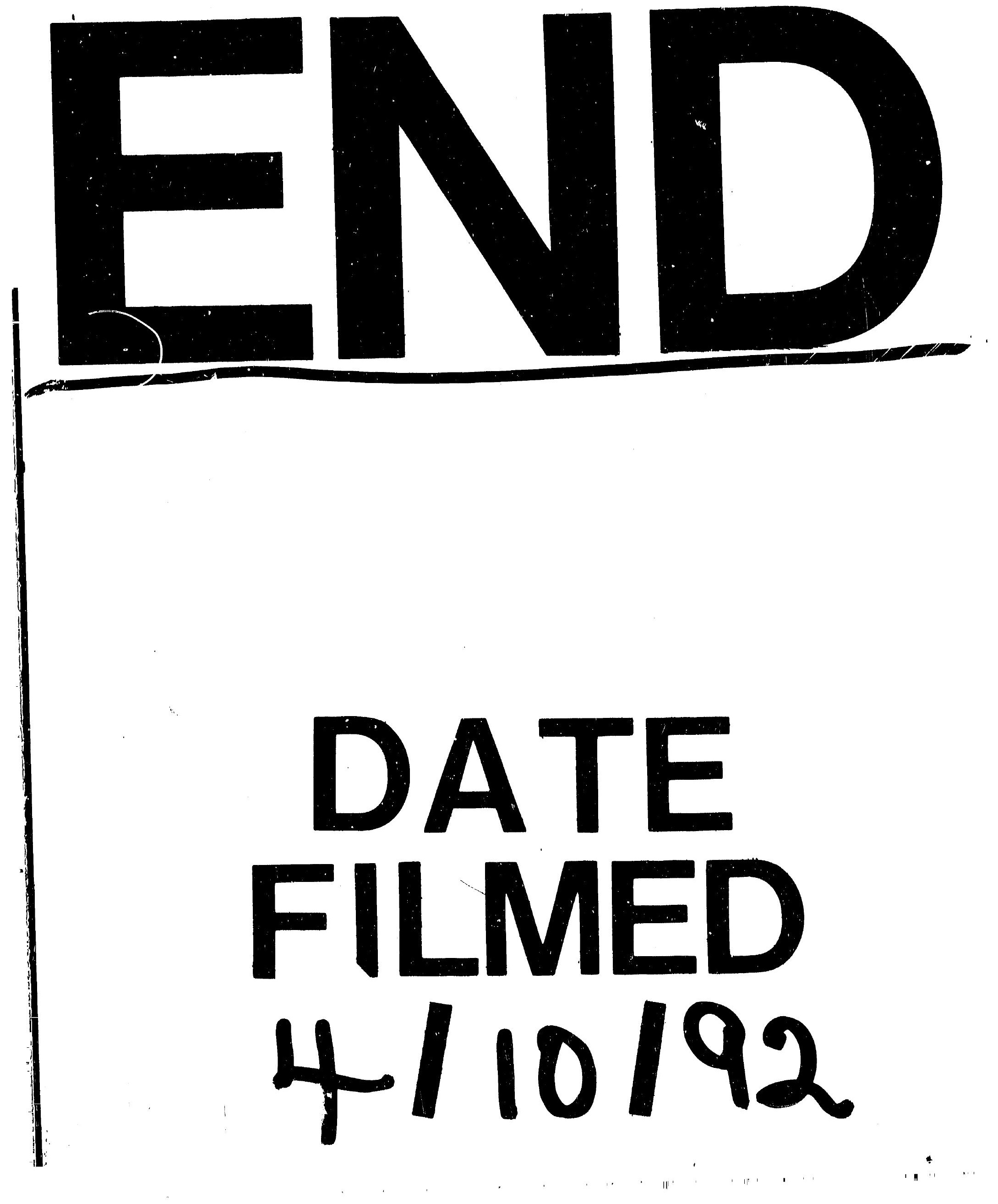


